\title{
Identification of Resistance of Guangxi Oryza Rufipogon to Sogatella Furcifera with Stem Evaluation Method
}

\author{
Guangxi University \\ xinying guo \\ Guangxi University \\ liang luo \\ China Agricultural University \\ kaichong teng \\ Guangxi University \\ yaoyu fang \\ Guangxi University \\ neng zhao \\ Guangxi University \\ fang liu \\ Guangxi University \\ baoxiang qin \\ Guangxi University \\ jijing luo \\ Guangxi University \\ rongbai li \\ Guangxi University
}

xuan wang ( $\nabla$ wangxuan01234@126.com )

\section{Research Article}

Keywords: Oryza rufipogon, Sogatella furcifera, stem evaluation method, resistance resources, regional distribution

Posted Date: October 27th, 2021

DOI: https://doi.org/10.21203/rs.3.rs-874060/v1

License: (a) (i) This work is licensed under a Creative Commons Attribution 4.0 International License. Read Full License 


\section{Abstract}

White-backed planthopper (WBPH) is a devastating pest of rice, which significantly reduces production and causes millions of dollars in losses worldwide annually. Screening of germplasm resources with resistance to white-backed planthopper plays a crucial role in crop insect resistance breeding. In the present research, a total of 1080 accessions of Guangxi common wild rice(Oryza rufipogon) was screened for WBPH resistance through stem evaluation method (SEM). After preliminary screening, 49 resistance resources were identified, accounting for $4.54 \%$ of the total. After verified identification at the adult stage, 15 resistance resources with stable resistance to white-backed planthopper were finally obtained as resistant resources. The regional distribution analysis showed that the highest frequency of WBPH resistance resources appeared in Fusui county located in the upper reaches of Xijiang river(6.80\%). Meanwhile, the highest frequency of the high resistance (HR and R) resources appeared in Pingnan located in lower reaches(2.79\%). The 49 resistant accessions were genotyped for 43 SSR markers evenly distributed on the 12 chromosomes. The genetic diversity analysis showed a high polymorphism level of common wild rice populations. Fusui county possesses higher level of genetic diversity than the Yongning district and Pingnan county populations. The results from this study are essential to analyze resistance genes, cultivate insect-resistant varieties, and provide regional guidance for the exploration of resistance resources in the future.

\section{Introduction}

White-backed planthopper(Sogatella furcifera) is one of the main pests in rice production, which distributes widely in major rice areas in the world. It not only directly sucks the phloem sap of rice plants, but also spreads the virus to harm the growth of rice, which has become an important factor restricting the stable production of rice (Shen et al. 2003). Since the introduction of high-yielding indica rice in the 1970s, the prevalence of white-backed planthopper has become more and more serious(Sogawa et al. 2003; Zhou et al. 2013). Chemical control is currently the main method for integrated control of WBPH, but it is expensive and not environmental friendly. At the same time, it also enhances the resistance of WBPH and promotes the resurgence of WBPH (Wang et al.2006). The practice shows that breeding resistant varieties is one of the most effective measures to prevent and control WBPH economically and environmentally.

Common wild rice (Oryza rufipogon Griff.), proved as the ancestor species of cultivated rice, has the AA genome just like the cultivated rice, which has important utilization value (Tang et al. 2008; Fuller et al. 2010). The genetic diversity of common wild rice has been widely studied in recent years. Lam(2019) studied centers of nuclear and maternal diversity of wild rice populations along the Mekong Delta, and illustrated Dong Thap population and Can Tho population had the highest and lowest genetic diversity, respectively. Shishido(2019) evaluated genetic diversity and genetic structure of wild rice populations in seven research sites in Myanmar, and found that these populations retained different levels of genetic diversity. Sandamal (2018) studied 11 natural populations of 0 . rufipogon and $O$. nivara from various environmental conditions in Sri Lanka, and found a moderate to high level of genetic structure diversity. 
Singh (2018) analyzed the genetic diversity of 418 O. rufipogon Griff. accessions collected from 64 districts of India and clarified the significant correlation between genetic and spatial distances of the accessions. Huang XH(2012) compared the common wild rice resources in different countries and provinces in China, and concluded that Guangxi is the genetic diversity center of common wild rice and the origin center of cultivated rice in China.

In the long-term evolution process of Guangxi wild rice, many excellent disease and insect-resistance traits were selected by natural selection (Hong et al. 2017; Qin et al. 2014; Wei et al. 2009; Li et al. 2006). Many valuable genes were discovered by researchers(Chen et al. 2009; Li et al. 2006; Yang et al. 2012; Wang et al. 2015; Li et al 2019) and has been gradually applied in breeding (Chen et al. 2010;Liu et al. 2011, 2013; Yang et al. 2010; Zhao et al. 2013). Therefore, it is believed that the exploration of resistant resources from common wild rice and the subsequent identification of resistance genes is of great significance for breeding resistant varieties to the control of WBPH.

In the past decades, the screening of a large number of different germplasm resources for resistance against WBPH have been carried out, and some achievements have been obtained. Qin (1993) identified the resistance of Guangxi wild rice to WBPH, and selected 18 resistant accessions. Li(1994) identified the resistance of 1236 O. rufipogon and 197 O. officinalis accessions to the WBPH through the seedling bulk test. The results showed that 218 accessions of $O$. rufipogon showed moderate resistance (MR), and all the 197 accessions of $O$. officinalis were resistant to the WBPH. Li (2003) screened 393 accessions of rice varieties for resistance to WBPH using standard seedbox screening, and discovered 76 resistant germplasm resources. Cheng(2007) screened 520 accessions of rice varieties from the International Network for Genetics Evaluation using seedling bulk test, and found 118 WBPH-resistant resources. Huang FK (2012) screened 383 accessions of rice for resistance to WBPH through seedling bulk test, and identified 108 resistant to WBPH.

Nevertheless, the number of resistance resources available for breeding is still limited. Also, the identification method for screening resistant resources needs to be improved, and the guidance area for exploring resistance resources is still unclear. In the present research, 1080 accessions of Guangxi wild rice were screened for resistance against WBPH by stem evaluation method, aiming to provide excellent resistant resources for exploring resistance genes and breeding resistant varieties. The analysis of the regional distribution and genetic diversity of resistant resources could provide guidance in exploring resistance resources in the future.

\section{Materials And Methods}

\subsection{Experimental accessions}

Tested accessions:1080 accessions of common wild rice germplasms from 3 major habitad regions in Guangxi were selected from the Germplasm Nursery of Wild Rice in Guangxi University. Among them, 397 accessions were from Fusui county of Chongzuo city, 468 from Yongning County of Nanning city, and 
215 from Pingnan county of Guigang city. Fusui, Yongning and Pingnan are located in the upper, middle and lower reaches of Xijiang river basin, respectively(Fig. 1). Taichung Native 1 cultivar (TN1) was chosen as the susceptible control. WBPH insects were collected from rice fields in Nanning and raised on TN1 to multiply enough number of nymphs before ready for infestation.

\subsection{Preliminary screening of resistant accessions}

The resistance evaluation of each 0 . rufipogon Griff. accession was carried out in the greenhouse during the spring season of 2018. The WBPH resistance of 1,080 accessions of common wild rice were subjected to preliminary screening with Stem Evaluation Method (SEM) described as Luo(2021). The resistance level of each stem was recorded according to the rating scale proposed by Luo(2021), and the average resistance score from three biological repeats represented the resistance level of each 0 . rufipogon Griff. accession.

\subsection{Re-screening of resistant accessions and confirmation of resistant resources}

The resistant accessions with the resistance level of scale 1 (highly resistant, HR) or scale 3 (resistant, R) obtained from the preliminary screening were subjected to re-screening at the adult stage. Stems of the selected resistant accessions were transplanted sparsely into a cement pool in the greenhouse, with the interval of each plant was $70 \mathrm{~cm} \times 70 \mathrm{~cm}$. The TN1 plants were planted in the middle as control. The pool maintained at $26{ }^{\circ} \mathrm{C}$ to $30{ }^{\circ} \mathrm{C}$, and shallow water. After about 5 weeks, when the plants grew up to adult stage, they were infested with second or third instar age of WBPH nymphs at a density of more than 50 nymphs per stem and covered by an insect-proof net. When the TN1 plants completely wilted, the resistance of the accessions to WBPH could be evaluated, and those with scale 1 (highly resistant, HR) or scale 3 (resistant, R) accessions were confirmed as resistant resources. The standard on which identification at adult-plant stage was based on the rating scale proposed by Luo (2021).

\subsection{Genetic diversity analysis of resistant accessions}

CTAB method was used to extract genomic DNA from common wild rice accessions (Chen and Ronald, 1999). Forty-three pairs of SSR markers (Table 3) with good polymorphism covering 12 chromosomes of rice were selected from SSR markers designed by Li(2015). The PCR was performed as the following program: initial denaturation at $95^{\circ} \mathrm{C}$ for $5 \mathrm{~min} ; 34$ cycles of denaturation at $95^{\circ} \mathrm{C}$ for $30 \mathrm{~s}$, annealing at $57^{\circ} \mathrm{C}$ for $30 \mathrm{~s}$ and extension at $72^{\circ} \mathrm{C}$ for $30 \mathrm{~s}$; and a final extension step at $72^{\circ} \mathrm{C}$ for $5 \mathrm{~min}$. The amplified products were detected by polyacrylamide gel electrophoresis (PAGE) with a concentration of $8 \%$, and the genotypes of each accession were recorded for genetic diversity analysis.

The average number of alleles $(\mathrm{Na})$, the effective number of alleles $(\mathrm{Ne})$, Shannon's information index $(\mathrm{I})$, actually observed heterozygosity $\left(H_{o}\right)$, expected observed heterozygosity $\left(H_{e}\right)$, diversity index $(\mathrm{Nei})$, and the polymorphism information content (PIC) was calculate through Popgene 32 (Yeh and Yang, 1999) and PIC-CALC (Nagy et al.2012). The cluster analysis was performed through MEGA-X. The principal 
component analysis $\left(\mathrm{PC}_{\mathrm{o}} \mathrm{A}\right)$ was performed through the online website ImageGP

(http://www.ehbio.com/imagegp/index.php/home/index/index.html). The population structure analysis of common wild rice resources was performed through the Structure 2.3.4 for three times. $\mathrm{K}$ value presented the population number, and was set to $1-10$.

\section{Results}

\subsection{Preliminary screening of WBPH resistant accessions}

The WBPH resistance of 1,080 accessions of Guangxi common wild rice was preliminarily screened with SEM (Fig. 2). The identification results indicated that when the susceptible variety TN1 fell into scale 9 (highly susceptible, HS), a total of 49 accessions of common wild rice showed different degrees of resistance to $\mathrm{BPH}$, accounting for $4.54 \%$ of the total. Results showed that there were 4 with scale $1(\mathrm{HR})$, 11 with scale $3(\mathrm{R}), 34$ with scale $5(\mathrm{MR})$, accounting for $0.37 \%, 1.02 \%$, and $3.15 \%$ of the total, respectively (Table 1). The accessions with scale 1 (HR) and scale $3(\mathrm{R})$ accounting for $1.39 \%$ of the total. It can be seen that there was a high proportion of WBPH resistant accessions in Guangxi common wild rice resources, which was ideal resources for exploring WBPH resistance genes and breeding WBPH resistant varieties (Table 1).

Table 1

Preliminary screening results of WBPH resistance of common wild rice origenated from three major habitads of Guangxi

\begin{tabular}{|c|c|c|c|c|c|c|c|c|}
\hline \multirow{3}{*}{$\begin{array}{l}\text { Resistance } \\
\text { scale }\end{array}$} & \multirow{3}{*}{$\begin{array}{l}\text { No. of } \\
\text { accessions } \\
\text { identified }\end{array}$} & \multirow{3}{*}{$\begin{array}{l}\text { Percentage of total } \\
\text { accessions }\end{array}$} & \multicolumn{6}{|c|}{ Distribution region } \\
\hline & & & \multicolumn{2}{|c|}{ Fusui } & \multicolumn{2}{|c|}{ Yongning } & \multicolumn{2}{|c|}{ Pingnan } \\
\hline & & & No. & $(\%)$ & No. & (\%) & No. & $(\%)$ \\
\hline $1(\mathrm{HR})$ & 4 & 0.37 & 0 & 0 & 0 & 0 & 4 & 1.86 \\
\hline $3(\mathrm{R})$ & 11 & 1.02 & 4 & 1.01 & 5 & 1.07 & 2 & 0.93 \\
\hline $5(\mathrm{MR})$ & 34 & 3.15 & 23 & 5.79 & 8 & 1.71 & 3 & 1.40 \\
\hline $7(S)$ & 289 & 26.76 & 87 & 21.91 & 165 & 35.26 & 37 & 17.21 \\
\hline $9(\mathrm{HS})$ & 742 & 68.70 & 283 & 71.28 & 290 & 61.97 & 169 & 78.60 \\
\hline total & 1080 & 100 & 397 & & 468 & & 215 & \\
\hline
\end{tabular}

\subsection{Re-identification and confirmation of resistant resources}

The 15 accessions with preliminary screening scales of 1 and 3 were selected as candidate accessions for WBPH highly resistant resources, and their stems were transplanted to the identification pool along with the susceptible check TN1 (Table 1). Five weeks after transplanting, re-identification was carried out 
with the method of excessive infestation to confirm their resistance to the WBPH at the adult stage. The results showed that when the TN1 plants completely wilted, the 15 highly resistant resources still showed stable high resistance, verifying the accuracy of the preliminary screening results (Fig. 3). Therefore, these 15 accessions were confirmed to be highly resistant resources(Table 2).

Table 2

Resistance and origins of the 15 accessions of WBPH-resistant common wild rice resources

\begin{tabular}{|llllll|}
\hline $\begin{array}{l}\text { Resistance } \\
\text { resources No. }\end{array}$ & $\begin{array}{l}\text { Resistance } \\
\text { scale }\end{array}$ & Origin & $\begin{array}{l}\text { Resistance } \\
\text { resources No. }\end{array}$ & $\begin{array}{l}\text { Resistance } \\
\text { scale }\end{array}$ & Origin \\
\hline 1300 & 1.2 & Pingnan & 287 & 3.3 & Yongning \\
\hline 1310 & 1.1 & Pingnan & 920 & 3.3 & Yongning \\
\hline 1325 & 1.3 & Pingnan & 1060 & 3.3 & Yongning \\
\hline 1331 & 1.2 & Pingnan & 370 & 3.2 & Fusui \\
\hline 1280 & 3.3 & Pingnan & 414 & 3.4 & Fusui \\
\hline 1297 & 3.2 & Pingnan & 500 & 3.2 & Fusui \\
\hline 276 & 3.3 & Yongning & 537 & 3.2 & Fusui \\
\hline 286 & 3.1 & Yongning & & & \\
\hline
\end{tabular}

\subsection{Geographic distribution of WBPH resistant accessions}

The 1080 common wild rice accessions tested in this research were collected from three major wild rice distribution regions: Fusui, Yongning, and Pingnan (Fig. 1).

Within the 397 accessions collected from Fusui distribution region, 4 accessions (1.01\%) showed scale 3 $(R)$ and 23 accessions (5.79\%) showed scale 5 (MR), respectively, which accounted for $6.8 \%$ of the total samples. Within the 468 accessions collected from Yongning distribution region, 5 accessions $(1.07 \%)$ showed scale $3(\mathrm{R})$ and 8 accessions $(1.71 \%)$ showed scale 5 (MR), respectively, which accounted for $2.78 \%$ of the total samples. Within the 215 accessions collected from Pingnan distribution region, 4 accessions (1.86\%) showed scale 1 (HR), 2 accessions ( $0.93 \%)$ showed scale $3(R)$ and 3 accessions $(1.40 \%)$ showed scale $5(M R)$, respectively, which accounted for $4.19 \%$ of the total samples.

The distribution of WBPH resistance resources in common wild rice showed that the highest frequency $(6.80 \%)$ is found in Fusui county in the upper reaches of Xijiang river basin, followed by Pingnan in the lower reaches $(4.19 \%)$ and Yongning district in the middle reaches $(2.78 \%)$. The distribution of resources with the high resistance (HR and R) in common wild rice showed that the highest frequency $(2.79 \%)$ was in Pingnan. The frequency difference between Fusui and Yongning was very small, which was $1.01 \%$ and $1.07 \%$ respectively (Table 1 ). 


\subsection{Genetic diversity of BPH resistant accessions}

Forty-three molecular markers were adopted to detect the genotype of the 49 common wild rice WBPH resistant resources obtained in this study (Table 3). All the molecular markers showed polymorphism, and the number of polymorphic loci reached $100 \%$. The actual number of alleles $(\mathrm{Na})$ detected by markers were 2 to 4 , with 3.0465 on average. The expected number of alleles (Ne) ranged from 1.5606(RM14) to 3.5809(RM218), with an average of 2.3512. The Shannon's Information index (I) varied from 0.5449 (RM14) to 1.3159 (RM218), with an average of 0.9247 . The diversity index (Nei) varied from 0.38 (RM525 and RM4801) to 0.7207(RM218), with an average value of 0.5606. The expected observed heterozygosity $\left(\mathrm{H}_{\mathrm{e}}\right)$ ranged from $0.384(\mathrm{RM} 525$ and RM4801) to $0.7282(\mathrm{RM} 218)$, with an average of 0.5664. The actual observation heterozygosity $\left(H_{0}\right)$ ranged from $0.0204(R M 277)$ to 0.9592 (RM264 and RM484), with an average of 0.58 . The polymorphic information content (PIC) ranged from 0.2947(RM14) to 0.6172 (RM17), with an average value of 0.4828 , close to 0.5 (Table 3 ). All this indicated that this batch of resistant sources had rich genetic diversity, and enough attention should be given to protect and utilize the resistant resources. 
Table 3

Sequences of 43 SSR Primers used for genetic diversity analysis of resistant resources.

\begin{tabular}{|llllllll|}
\hline Locus & na & ne & Obs_Het & Exp_Het & Nei & I & PIC \\
\hline RM1 & 3 & 2.5407 & 0.1633 & 0.6127 & 0.6064 & 1.0126 & 0.5378 \\
\hline RM14 & 2 & 1.5606 & 0.4694 & 0.3629 & 0.3592 & 0.5449 & 0.2947 \\
\hline RM17 & 4 & 3.1041 & 0.3878 & 0.6848 & 0.6778 & 1.2254 & 0.6172 \\
\hline RM23 & 3 & 2.3986 & 0.8367 & 0.5891 & 0.5831 & 0.9482 & 0.4947 \\
\hline RM31 & 4 & 2.4676 & 0.3469 & 0.6009 & 0.5948 & 1.0925 & 0.541 \\
\hline RM91 & 3 & 2.3254 & 0.7551 & 0.5758 & 0.57 & 0.9454 & 0.4944 \\
\hline RM101 & 3 & 2.0513 & 0.2245 & 0.5178 & 0.5125 & 0.8836 & 0.4587 \\
\hline RM103 & 3 & 2.7519 & 0.5918 & 0.6432 & 0.6366 & 1.0529 & 0.5625 \\
\hline RM107 & 3 & 2.9015 & 0.4082 & 0.6621 & 0.6554 & 1.0821 & 0.5817 \\
\hline RM110 & 3 & 1.6496 & 0.449 & 0.3979 & 0.3938 & 0.6615 & 0.3367 \\
\hline RM156 & 3 & 2.122 & 0.7347 & 0.5342 & 0.5287 & 0.8083 & 0.4177 \\
\hline RM188 & 3 & 2.6722 & 0.6122 & 0.6322 & 0.6258 & 1.0312 & 0.5476 \\
\hline RM193 & 3 & 2.2058 & 0.9796 & 0.5523 & 0.5466 & 0.8588 & 0.4431 \\
\hline RM201 & 3 & 2.2748 & 0.3061 & 0.5662 & 0.5604 & 0.9154 & 0.4757 \\
\hline RM212 & 4 & 2.3574 & 0.2653 & 0.5817 & 0.5758 & 1.0755 & 0.5292 \\
\hline RM216 & 3 & 2.7253 & 0.7755 & 0.6396 & 0.6331 & 1.0428 & 0.555 \\
\hline RM218 & 4 & 3.5809 & 0.4082 & 0.7282 & 0.7207 & 1.3159 & 0.667 \\
\hline RM228 & 3 & 2.216 & 0.5918 & 0.5544 & 0.5487 & 0.8847 & 0.4576 \\
\hline RM229 & 3 & 1.701 & 0.2857 & 0.4164 & 0.4121 & 0.7429 & 0.375 \\
\hline RM234 & 3 & 2.4714 & 0.8163 & 0.6015 & 0.5954 & 0.9987 & 0.529 \\
\hline RM237 & 3 & 2.2492 & 0.8367 & 0.5611 & 0.5554 & 0.8808 & 0.455 \\
\hline RM242 & 3 & 2.1126 & 0.9388 & 0.5321 & 0.5267 & 0.8062 & 0.4166 \\
\hline RM256 & 3 & 1.9802 & 0.7755 & 0.5001 & 0.495 & 0.7277 & 0.3822 \\
\hline RM257 & 3 & 2.1563 & 0.5714 & 0.5418 & 0.5362 & 0.871 & 0.4501 \\
\hline RM264 & 3 & 2.0815 & 0.9592 & 0.5249 & 0.5196 & 0.7784 & 0.4042 \\
\hline RM276 & 3 & 1.8569 & 0.4286 & 0.4662 & 0.4615 & 0.7935 & 0.4058 \\
\hline
\end{tabular}




\begin{tabular}{|llllllll|}
\hline Locus & na & ne & Obs_Het & Exp_Het & Nei & I & PIC \\
\hline RM281 & 3 & 2.0601 & 0.551 & 0.5199 & 0.5146 & 0.7733 & 0.4015 \\
RM286 & 3 & 2.614 & 0.6531 & 0.6238 & 0.6175 & 1.0145 & 0.5365 \\
RM346 & 3 & 2.5128 & 0.6122 & 0.6082 & 0.602 & 1.008 & 0.5348 \\
RM425 & 3 & 2.7886 & 0.9184 & 0.648 & 0.6414 & 1.0604 & 0.5673 \\
\hline RM429 & 3 & 2.6183 & 0.3265 & 0.6244 & 0.6181 & 1.0188 & 0.5397 \\
\hline RM484 & 3 & 2.5394 & 0.9592 & 0.6125 & 0.6062 & 1.0011 & 0.5287 \\
\hline RM500 & 3 & 2.2502 & 0.7755 & 0.5613 & 0.5556 & 0.881 & 0.4552 \\
\hline RM515 & 3 & 2.7176 & 0.551 & 0.6385 & 0.632 & 1.0481 & 0.5597 \\
\hline RM525 & 2 & 1.613 & 0.5102 & 0.384 & 0.38 & 0.5679 & 0.3078 \\
\hline RM566 & 4 & 2.5102 & 0.898 & 0.6078 & 0.6016 & 1.0254 & 0.5255 \\
\hline RM567 & 3 & 1.9901 & 0.551 & 0.5026 & 0.4975 & 0.7922 & 0.4079 \\
\hline RM577 & 3 & 2.905 & 0.4286 & 0.6625 & 0.6558 & 1.0828 & 0.5822 \\
\hline RM1178 & 3 & 2.4413 & 0.5918 & 0.5965 & 0.5904 & 0.9874 & 0.5215 \\
\hline RM4801 & 2 & 1.613 & 0.5102 & 0.384 & 0.38 & 0.5679 & 0.3078 \\
\hline RM5473 & 3 & 2.5943 & 0.3061 & 0.6209 & 0.6145 & 1.0217 & 0.5428 \\
\hline RM8209 & 3 & 2.3516 & 0.8571 & 0.5807 & 0.5748 & 0.951 & 0.4978 \\
\hline Mean & 3.0465 & 2.3512 & 0.58 & 0.5664 & 0.5606 & 0.9247 & 0.4828 \\
\hline
\end{tabular}

$\mathrm{Na}$, actual number of alleles; Ne, expected number of alleles; I, Shannon's Information index; Obs_Hom:actual observation homozygosity;Exp_Hom:expected observed homozygosity;Obs_Het:actual observation heterozygosity; Exp_Het:expected observed heterozygosity;Nei, diversity index; PIC, polymorphic information content

The difference analysis of genetic diversity of WBPH resistant accessions in the three regions was conducted, and results revealed that the highest genetic diversity index was found in Fusui county, followed by Yongning district, and Pingnan county was the lowest(Table 4). 
Table 4

genetic diversity differences among WBPH resistant resources in three distribution areas

\begin{tabular}{|llllllll|}
\hline Origin & na & ne & Obs_Het & Exp_Het & Nei & I & PIC \\
\hline Fusui & 2.9302 & 2.2988 & 0.5788 & 0.5630 & 0.5526 & 0.8968 & 0.4699 \\
\hline Yongning & 2.6744 & 2.1231 & 0.5689 & 0.5207 & 0.5007 & 0.8067 & 0.4347 \\
\hline Pingnan & 2.4884 & 2.0186 & 0.5995 & 0.4978 & 0.4701 & 0.7440 & 0.4119 \\
\hline
\end{tabular}

$\mathrm{Na}$, actual number of alleles; Ne, expected number of alleles; I, Shannon's Information index [Lewontin (1972)]; Obs_Hom:actual observation homozygosity;Exp_Hom:expected observed homozygosity;Obs_Het:actual observation heterozygosity; Exp_Het:expected observed heterozygosity;Nei, diversity index; PIC, polymorphic information content

\subsection{Cluster analysis of WBPH resistant accessions}

The genetic distance of Nei calculated by POPGENE was used to draw the cluster diagram among accessions in WBPH resistant population by Mega software. The 49 WBPH-resistant accessions could be divided into three clusters (Fig. 4). The first cluster had the most genetic diversity, with 7 WBPH resistant accessions from Fusui county, including GXU wild rice accession numbers 431, 425, 434, 421, 464, 449 and 418. The second cluster contained 21 resistant accessions, composed of resistant resources from three distribution areas. The third cluster contained 21 resistant accessions, composed of those from Fusui county and Yongning district. The second and the third clusters were both divided into two subgroups, cluster 2 - 1 contained all the resistant accessions from Pingnan county, the rest were resistant accessions from Yongning district, and cluster3-1 was composed of all the resistant accessions from Fusui county. In these five clusters, the resistant accessions in the three regions did not form independent clusters. The accessions showed intersecting among the three regions. The resistant accessions from Fusui county had the most diverse genetic distances, which were distributed in three clusters. The resistant accessions from Yongning district were distributed in two clusters. The most limited distribution of resources diversity was in Pingnan county, only in the second cluster. The most abundant genetic diversity of resistance resources was that of Fusui county, followed Yongning district, and finally Pingnan county. This finding was consistent with the results of genetic diversity analysis (Fig. 4).

\subsection{PCoA Analysis of WBPH Resistant Accessions}

In order to verify the cluster analysis results of WBPH resistant accessions, $\mathrm{PC}_{0} \mathrm{~A}$ analysis was conducted using ImageGP online website. In the two-dimensional coordinate chart, the first principal component $\left(P C_{0} A 1\right)$ was used as the abscissa, and the second principal component $\left(P C_{0} A 2\right)$ was used as the ordinate. In the chart, the distance between the resistant accessions was related to the genetic relationship. According to the $\mathrm{PC}_{0} \mathrm{~A}$ analysis of the origins of resistant accessions, it can be divided into three groups. The first group was composed of accessions from Fusui county. The second group 
contained accessions from three distribution areas, of which the accessions from Yongning district were closer to that from Pingnan county. The third group was composed of resistant accessions from Fusui county and Yongning district. These results indicated that the resistant accessions were not completely classified according to geographical origin, and there was a phenomenon of intersecting. The resistant accessions from Fusui county were the most widely distributed in the chart, followed by that from Yongning district, and finally Pingnan county, indicating that the genetic diversity of resistant accessions from different origins was in the order of Fusui county $>$ Yongning district $>$ Pingnan county. These results are consistent with the results of cluster analysis (Fig. 5).

\subsection{Population structure analysis of WBPH resistant accessions}

Based on the Structure 2.3.4 model, the log-likelihood LnP (D) showed an increasing trend with the increase of $\mathrm{K}$ value, and no obvious maximum inflection point showed in Fig. 6A. In further calculation of the $\Delta K$ value use the $L n P(D)$, the maximum inflection point appeared when $K=2$ (Fig. 6B). It could be concluded that the 49 WBPH resistant accessions in common wild rice could be divided into two stable clusters, and according to $\mathrm{K}$ value. The entire population could be divided into 2 potential genetic branches, that is, there were 2 subpopulation structures in this population (Fig. 6C). This potential differentiation would be a prediction of the future growth, breeding, and evolution of this batch of resistant resources. Based on the analysis of geographical sources, it was found that there was a phenomenon of mutual penetration among resistant resources in three different regions in terms of geographical origin. The genetic relationships of some accessions from different geographical origins were relatively close, indicating that the population structure had become diversified and the genetic basis was broad.

\section{Discussion}

White-backed planthopper (WBPH) is one of the main pests of rice, which spreads all over the rice area in China and seriously threatens the stable production of rice (Shen et al. 2003). The lack of resistant varieties is one of the main reasons for the epidemic of WBPH. According to the Chinese National Rice Data Center, The database for Chinese rice varieties and their genealogy, among 828 rice varieties approved by the Ministry of Agriculture and Rural Affairs of China(MARA) in 2017-2019, only 2 varieties, one three-line hybrid rice Quanyouhuazhan and one two-line hybrid rice C Liangyou 810, were found with resistance to WBPH and their resistance performance just reached moderate resistance (MR) level (http://www.ricedata.cn/variety/). Therefore, screening the germplasm resources against WBPH for breeding utilization is the key solution to solve the severe problem of WBPH in some areas in China.

At present, the standard seedbox screening test (SSST) method at seedling stage is mainly used to screen rice germplasm resources for resistance to WBPH (Li and Qin 1994; Cheng 2007; Huang FK et al. 2012). However, the operation of SSST is limited by experimental conditions due to the great demand for 
plants and insects. In addition, it is not suitable to infect excessive pest in identification at the seedling stage. Thus, SSST has the defects of long identification period and low accuracy, and is not ideal for screening stable resistant sources. In order to screen the wild rice for WBPH resistance more efficiently and accurately, the SEM method was used. In this method, a stem represents a plant. The amount of biomass of a stem is much smaller compared with a whole plant, with reduced insect demand and shortened evaluation period. With the limited number of WBPH insects raised for identification, SEM method allows more screening experiments and repeated identification. Combined the verification at the adult stage, it is more efficient and precise in determining stable resistant resources. Therefore, SEM has a good application prospect in screening resistant germplasm resources, constructing mapping population of resistance genes and breeding resistant varieties.

Common wild rice genome contains rich insect-resistant resources, and belongs to the same AA genome as cultivated rice, which has rich genetic diversity and great breeding value ( $\mathrm{Li}$ et al. 2006; Tang et al. 2008). Therefore, our research group has long been committed to exploring the rice planthopper resistant resources of common wild rice, including more than 30 resources with stable resistance to brown planthopper, and discovered 10 resistance genes, including bph19(t), bph20(t),bph21(t), bph22(t), bph23(t), Bph24(t), Bph27, Bph29, Bph35 and Bph36(Li et al. 2001; Li et al. 2006; Yang et al. 2012;Chen et al. 2009; Wang et al. 2015;Zhang et al. 2020; Li et al 2019).

In this study, 1080 accessions of Guangxi common wild rice from Fusui in the upper reaches of Xijiang river, Yongning in the middle reaches and Pingnan in the lower reaches of Xijiang river were screened for resistance to WBPH by SEM, and 49 resistant resources were identified, accounting for $4.54 \%$ of the total. The 49 resistant resources were repeatedly identified with SEM and verified at the adult stage, 15 resources with stable resistance were obtained as the resistance resources. These 15 resistance resources will enrich the genetic basis of resistance to WBPH, and can be used as candidate materials for exploring WBPH resistance genes and breeding WBPH resistant varieties.

According to the analysis of the geographic region and distribution of WBPH resistant accessions, Fusui county had the highest frequency of resistance resources (6.80\%), followed by Pingnan county (4.19\%), and Yongning district had the lowest resistance frequency (2.78\%). The frequency analysis of resistant resources in each area showed that the frequency at Pingnan county was the highest $(2.79 \%)$, while the frequency difference between Fusui county and Yongning district was very small, which was $1.01 \%$ and $1.07 \%$ respectively (Table 1 ). Fusui county with high amount and Pingnan county with high frequency of resistance resources to WBPH will be the key areas of common wild rice materials exploration.

The genetic diversity of wild rice has been widely studied by researchers all over the world. However, the genetic diversity of common wild rice in Guangxi has not been thoroughly elucidated. Xue(2016) studied the enter of genetic diversity and core collection of common wild rice, $O$, rufipogon Griff., in Guangxi, and found that the main sources of the core collection samples were regional populations from Yongning and Fusui, which were the most important populations of Guangxi common wild rice. But it is not clear how the genetic structure of accessions against WBPH distributed among these populations. In this study, we 
found that the genetic diversity of resistant accessions from different origins was in the order of Fusui county $>$ Yongning district $>$ Pingnan county. The geographical origin of the resistant resources mutual penetrated with each other in three regions. As shown in Fig. 6C, the entire population was divided into 2 potential genetic branches. The accessions from Pingnan county were all clustered in branch 1 , and the accessions of Yongning and Fusui were distributed in both branches respectively. The reason for this phenomenon may be that Fusui and Yongning are located in the upper and middle reaches of Xijiang river Basin, which are adjacent to each other and have relatively higher similarity of diversity, while Pingnan is located in the lower reaches of Xijiang river Basin far away from the former two origins geographically, hence presented larger difference (Fig. 3).

These results suggested that Guangxi common wild rice was an ideal material for collecting germplasm resources with resistance to WBPH and provided a guide for the future exploration of WBPH resistant germplasm resources.

\section{Conclusion}

The wild rice in Guangxi is an ideal material for exploring the resistance resources against white-backed planthopper. The identified resistance resources can be used to explore resistance genes and cultivate insect-resistant varieties. In the present research, 1080 accessions of Guangxi common wild rice were screened for resistance to WBPH by SEM. After repeated identification, 15 resistant resources were identified, which provided excellent material basis for exploring resistance genes and breeding resistant varieties. Stem evaluation method has the characteristics of high efficiency and accuracy, and has a bright application prospect in screening resistance resources, cultivating resistant varieties and constructing mapping population of resistance genes. Based on the analysis of the regional distribution of the resistant resources, it is suggested that Fusui county and Pingnan county should be focused on exploring the resistant germplasm resources of WBPH in the future.

\section{Declarations}

\section{Conflict of interest}

The authors declare that there is no conflict of interest.

\section{Funding}

This research was funded by Science and Technology Project of Guangxi(No.GuikeAB16380093)

\section{References}

1. Chen Y Z, Wei Y P, Li X Q,Chen Q, Sun R K,Yang L,Huang F K,Wei S M,Liu P Q,Huang D H,Zhang Y $X, L i, R . B .2009$. Evaluation and utilization of resistant sources to brown planthopper in rice[J]. 
Southwest China Journal of Agricultural Sciences .22(6),1581-1588.

2. Chen $Y$ Z,Chen Q,Sun $R$ K,Yang L,Huang F K,Huang D H,Wei S M,Zhang Y X,Liu P Q,Li R B.2010.Improvement of rice resistance to brown planthoppers[J].Southwest China Journal of Agricultural Sciences.23(4)1099-1106.

3. Chen, D.H., and P.C. Ronald. 1999. A rapid DNA minipreparation method suitable for AFLP and other PCR applications. Plant Mol. Biol. Rep. 17(1): 53-57. doi:10.1023/ A:1007585532036

4. Cheng $Z X, 2007$.Indentification, resisitance mechanisms and core collection of resisitant rice germplasms against whitebacked planthopper[D].Nanning:Guangxi University,1-61.

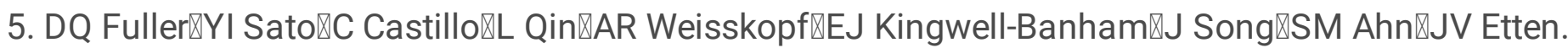
(2010). Consilience of genetics and archaeobotany in the entangled history of rice. Archaeological \& Anthropological Sciences, 2(2), 115-131.

6. Hong D W,Zhao Y,Luo D J,Shi L J,Li R B,Liu F.2017.Screening of broad-spectrum resisitant resources against rice bacterial leaf streak.[J]. Journal of Southern Agricultural .48(2):272-276.

7. Huang F K,Huang S S,Wu B Q,Wei S M.2012.Screening of rice germplasms resistant to brown planthopper(BPH) and white-backed planthopper(WBPH)[J].Plant Protection.38(04): 152-155.

8. Huang X H,Kurata N,Wang Z X,Wang A,Zhao Q,Zhao Y,Liu K Y,Lu H Y,Li W J,Guo Y L,Lu Y Q,Zhou C C,Fan D L,Weng Q J,Zhu Ch R,Huang T,Zhang L,Wang Y Ch,Feng L,Furuumi H,Kubo T,Miyabayashi T,Yuan X P,Xu Q,Dong G J,Zhan Q L,Li C Y,Fujiyama A,Toyoda A,Lu T T,Feng Q,Qian Q,Li J Y,Han B.2012.A map of rice genome variation reveals the origin of cultivated rice[J].Nature.490(7421) : 497-501.

9. Lam, D. T., Buu, B. C., Lang, N. T., Toriyama, K., Nakamura, I., \& Ishikawa, R. (2019). Genetic diversity among perennial wild rice Oryza rufipogon Griff., in the Mekong Delta. Ecology and evolution, 9(5), 2964-2977.

10. Li R B, Qin X Y, Wei S M ,Pandey M P,Pathak P K,Huang F K,Li Q,Luo Sh Y. Inheritance of resistance to brown planthopper in an Oryza rufipogon (Griff.)-derived line in rice[J]. Current science, 2001, 80(11):1421-1423.

11. Li R B,Li L S,Wei S M,Wei Y P,Chen Y Z,Bai DL,Yang L,Huang F K,Lv W L,Zhang X J,Li X Y,Yang X Q,Wei $Y$ W.2006. The evaluation and utilization of new genes for brown planthopper resistance in wild rice (Oryza rufipogon Griff.)[J] . Molecular Plant Breeding.4(3)365-371.

12. Li R B,Qin X Y.1994. The main finding of resistant identificatin of Guangxi wild rice to diseases and pests[J]. Guangxi Sciences.1(1):83-85.

13. Li X M.2003.Screening,genetic analysis and gene mapping of new resistant gene in rice towhitebacked planthopper,Sogatella furcifera[D].Nanjing:Nanjing Agricultural University,1-71.

14. Li Z H,Xue Y X,Zhou H L,Li Y,Usman B,Jiao X Z,Wang X Y.Liu F,Qin B X,Li R B.Qiu Y F.2019.Highresolution mapping and breeding application of a novel brown planthopper resistance gene derived from wild rice(Oryza rufipogon Griff)[J].Rice.12(41)1-13.

15. Li, M.B., H. Wang, and L.M. Cao. 2015. Evaluation of population structure, genetic diversity and origin of northeast asia weedy rice based on simple sequence repeat markers. Rice Sci. 22(4): 180-188. 
16. Liu K Y,Lu S N,Qiu J L,Li X Q,Ma Z F,Chen Q,Li C,Wei S M,Huang F K,Zhang Y X,Liu C,Huang D H,Li R B.2011.Development of brown planthopper resistance gene-inserted and accumulated lines of rice restorers [J]. Molecular Plant Breeding, 9(4), 410-417.

17. Liu K Y,Zhang Y X,Liu F,Qiu Y F,Feng J X,Huang F K,Wu B Q,Li Z F,Chen Y,Wei S L,Cen Z L,Qin B X,Wei M H,Wei Z,Li R B.2013.Resistant performance of bacterial blight and brown planthopper resisitance gene-pyramided lines in rice[J]. Southwest China Journal of Agricultural Sciences,26(05): 1852-1857.

18. Liang Luo, Xuan Wang, Xinying Guo, Taian Lu, Ruizhi Yuan, Shanyue Liao, Suosheng Huang, Yue Han, Babar Usman, Baoxiang Qin, Jijing Luo, Fang Liu, Fengkuang Huang, Rongbai Li. (2021). Evaluation of guangxi common wild rice (Oryza rufipogon Griff.) for resistance to brown planthopper (Nilaparvata lugens) using a new stem evaluation method. Crop Science.61(4): 2579-2592

19. Nagy, S., P. Poczai, I. Cernák, A.M. Gorji, G.Hegedűs, and J. Taller. 2012. PICcalc: an online program to calculate polymorphic information content for molecular genetic studies. Biochem. Genet. 50: 9-10. doi:10.1007/s10528-012-9509-1

20. Qin B X ,Liu C ,Jiu X Z,Huang D H,Zhang Y X,Ma Z F,Feng J X,Cen Z L,Liu F,Qiu Y F,Li R B.2014. Identification and evaluation of Guangxi wild rice (Oryza rufipogon Griff) resources with wide spectrum resistance tobacterial blight[J]. Journal of Southern Agriculture. 45(9):1527-1531.

21. Qin X Y,Li R B,Lin D H,Chen B L,Hu H.1993.Preservation,identification of Guangxi and utilization of wild rice germplasms resources in Guangxi. Guangxi Agricultural Sciences,(3):100-103.

22. Sandamal, S., Tennakoon, A., Meng, Q. L., Marambe, B., Ratnasekera, D., Melo, A., \& Ge, S. (2018). Population genetics and evolutionary history of the wild rice species Oryza rufipogon and O. nivara in Sri Lanka. Ecology and evolution, 8(23), 12056-12065. https://doi.org/10.1002/ece3.4665

23. Shen J H,Shang J M,Liu G J. 2003. Management of the whitedbacked planthoper,Sogatella furcifera:A mini-review[J]. Chinese Journal of rice science.(S1): 12-27.

24. Shishido R, Akimoto M, Htut T, Sato T, Ishii T. Assessment of genetic diversity and genetic structure of wild rice populations in Myanmar. Breed Sci. 2019;69(3):471-477. doi:10.1270/jsbbs.18165

25. Singh B, Singh N, Mishra S, Tripathi K, Singh BP, Rai V, Singh AK and Singh NK (2018) Morphological and molecular data reveal three distinct populations of Indian wild rice Oryza rufipogon Griff. species complex. Front. Plant Sci. 9:123. doi: 10.3389/fpls.2018.00123

26. Sogawa K,Liu G J,Teng K,Lin H F,Shen L L.2003.Resistance to the whitebacked planthopper,Sogatella furcifera in chinese japonica rice Chunjiang 06[J].Chinese Journal of rice science,(S1): 61-71.

27. Tang S X,Wei X H,Xu Q.2008.Progress of evaluation and utilization of wild rice resources abroad[J]. Journal of Plant Genetic Resources. 9 (2 ):223 -229.

28. Wang $Y H$,Wang M H.2006.Research progress on Nilaparvata lugens insecticide resistance and proliferation of the resistant biotype[J] .Agrochemicals ,(04): 227-230.

29. Wang Y,Cao L M,Zhang Y X,Cao C,Liu F,Huang F K,Qiu Y F,Li R B,Lou X J. 2015.Map-based cloning and characterization of bph29, a B3 domain-containing recessive gene conferring brown planthopper resistance in rice[J]. Journal of Experimental Botany.66(9),6035-6045. 
30. Wei Y P,Huang D H,Chen Y Z,Liu C,Yang L,Luo X M,Ma Z F,Zhang Y X,Liu Y L,Yang X Q,Li R B.2009.Identification and evaluation of resistance to Rice Blast in Guangxi wild rice resources[J]. Chinese Journal of rice science,23(04): 433-436.

31. Xue Yanxia, LIANG Yanli, FENG Xuan, HUANG Jinyan, Liu Fang, Qin Baoxiang, QIU Yongfu, Li Rongbai. Center of genetic diversity and core collection of common wild rice, Oryza rufipogon Griff., in Guangxi[J]. Journal of South China Agricultural University,2016,37(05):24-30.

32. Yang H N,Wei S L,Li X Q,Zhang Y X,Liu C,Chen Q,Cen Z L,Wei S M,Yang L,Huang F K,He W Q,Liu K Y,Huang D H,Ma Z F,Huang Y Y,Song J D,Li R B.2010.Rice pyramiding lines conferring resistance to brown planthopper and bacterial leaf blight by marker-assisted seletion[J].Molecular Plant Breeding,, 8(01): 11-19.

33. Yang L, Li R B, Li Y R, Huang F K,Chen Y Z,Huang Sh S,Huang F L,Liu ch,Ma F Z,Huang H D,Jiang J J. 2012.Genetic mapping of bph20(t) and bph21(t) loci conferring brown planthopper resistance to Nilaparvata lugens Stål in rice (Oryza sativa L.)[J]. Euphytica,183(2): 161-171.

34. Yeh, F.C., and R.C. Yang. 1998. POPGENE Version 1.31 Microsoft window-based freeware for population genetic analysis quick user guide. Univ. of Albert And Tim Boyle, Center for Intl. Forestry Res., 1-29.

35. Zhang Y X, Qin G, Ma Q Q, Wei M Y, Yang X H, Ma Z F, Liang H F, Liu C, Li Z J, Liu F, Huang D H, Li R B. 2020. Identification of a major locus Bph35 resisting to brown planthopper in rice (Oryza sativa L.) [J]). Rice science. 27(3):237-245

36. Zhao P,Feng R R,Xiao Q Z,Yang P Z,Liu P Q,Li R B.2013.Pyramiding brown planthopper genes, bph20(t)and bph21(t), and rice blast resistant gene Pi9 in rice (Oryza sativa L.)[J] . Jounral of Sounthern Agriculture. 44(6):885-892.

37. Zhou G, Xu D, Xu D, Zhang M. Southern rice black-streaked dwarf virus: a white-backed planthoppertransmitted fijivirus threatening rice production in Asia. Front Microbiol. 2013;4:270. doi:10.3389/fmicb.2013.00270

\section{Figures}




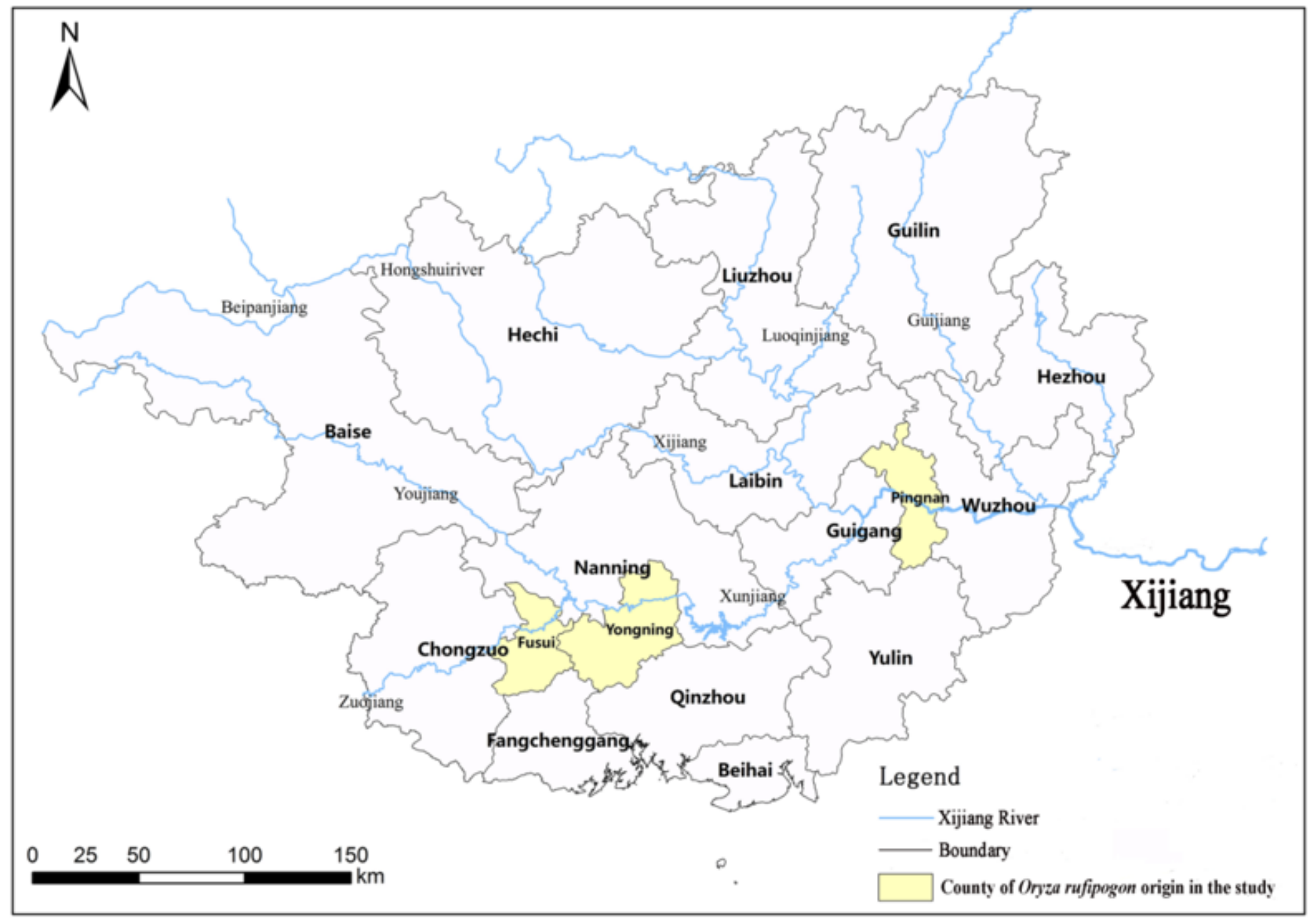

Figure 1

Geographical locations of 1080 0. rufipogon germplasms sampled in this study.
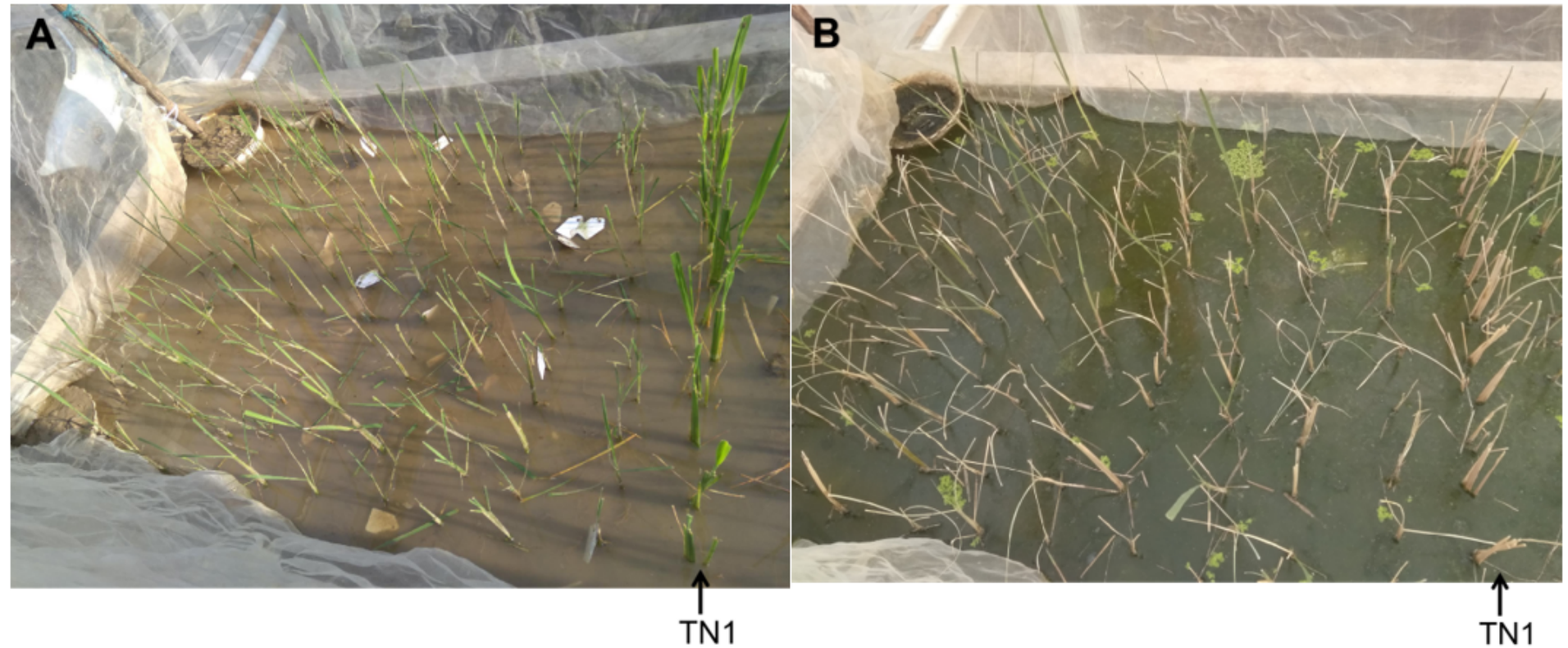

Figure 2 
Screening for WBPH resistance resources of Guangxi common wild rice by stem evaluation method

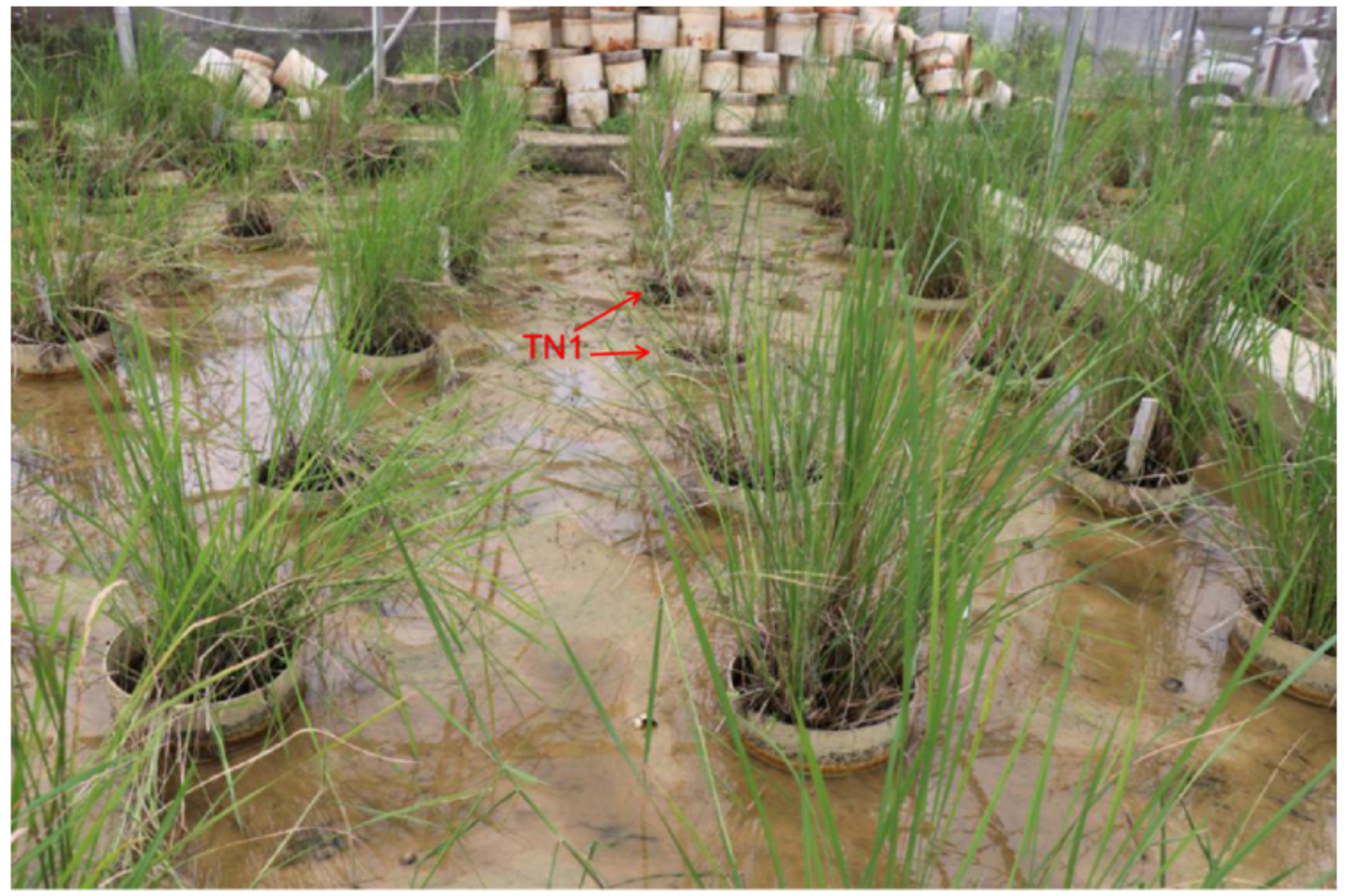

Figure 3

Re-identification of candidate accessions at the adult stage for WBPH resistant resources. 

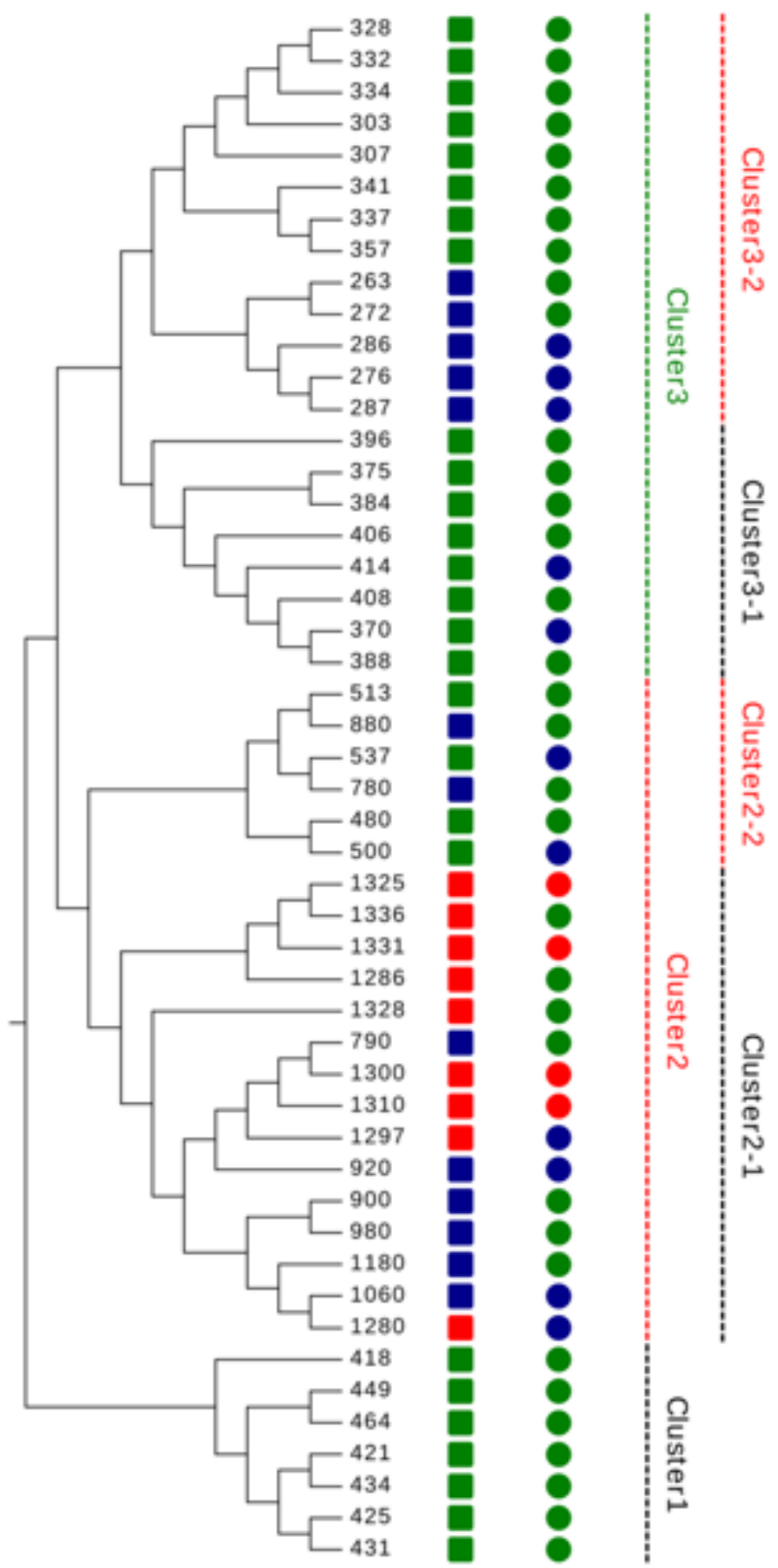

The numbers for the 49 accessions was GXU accession numbers of common wild rice of Guangxi University.

:Pingnan; $\square$ :Yongning; $\square$ :Fusui. $\mathrm{O}$ :Scale $1(\mathrm{HR})$;

:Scale 3(R); : Scale 5(MR).

\section{Figure 4}

UPHMA cluster analysis of $49 \mathrm{WBPH}$ - resistant accessions from common wild rice (0. rufipogion). 


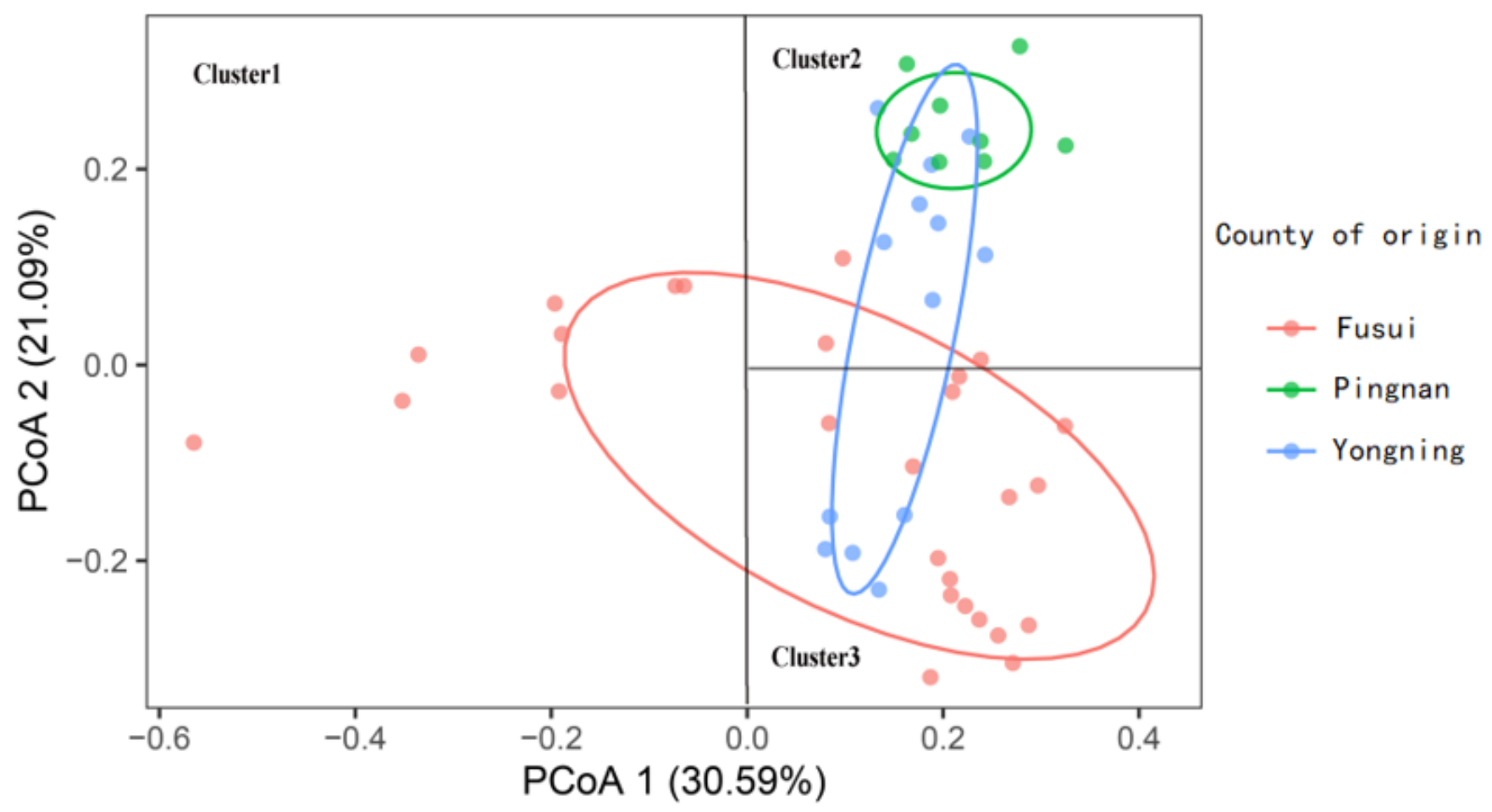

Figure 5

PCoA analysis of $49 \mathrm{WBPH}$ resistant accessions of common wild rice (O. rufipogon)

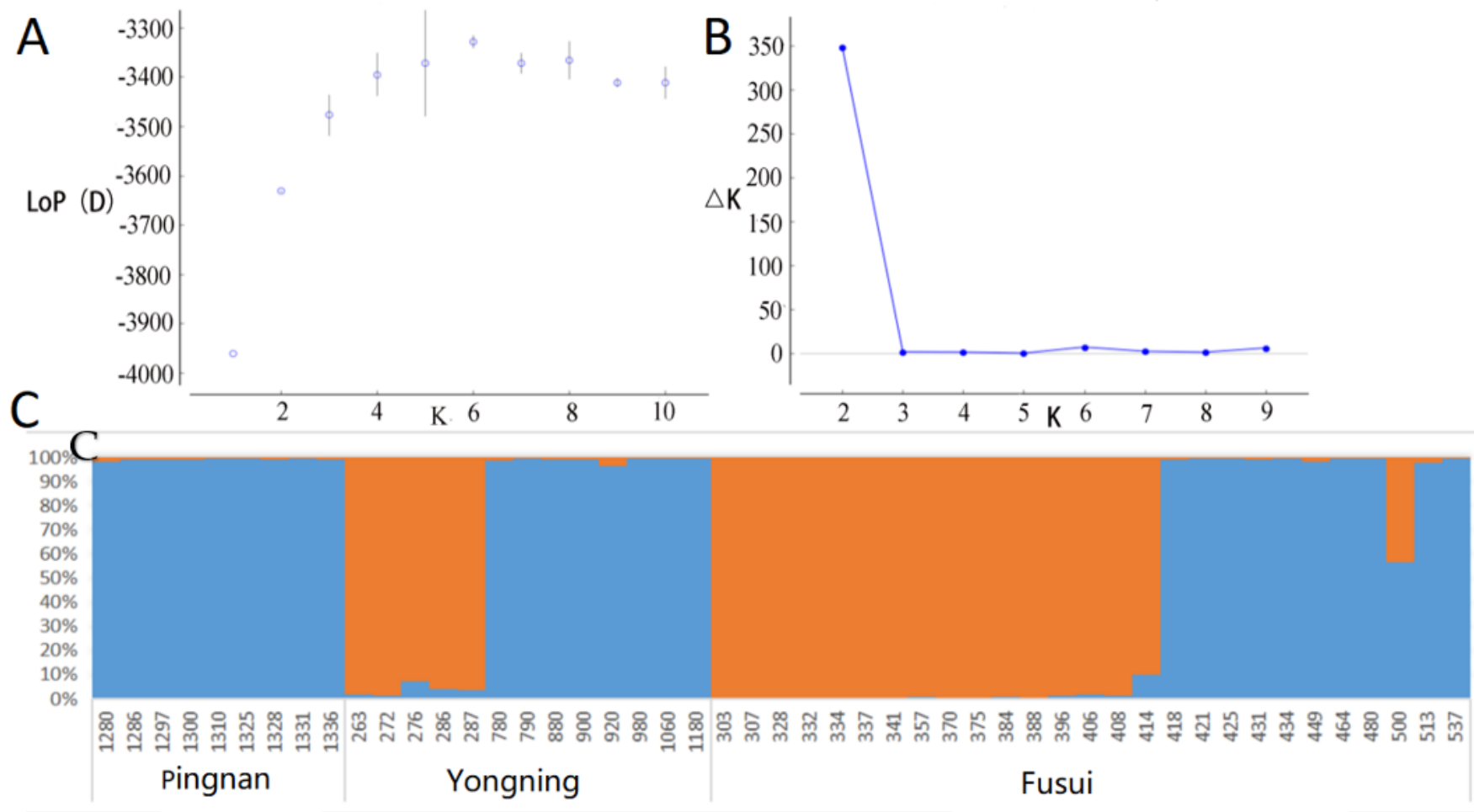

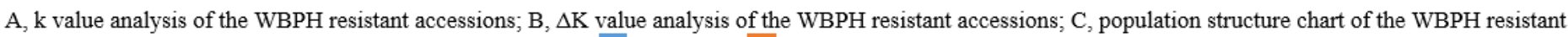
accessions based on $\Delta \mathrm{K}=2$ and arranged by county of origin, : Cluster 1, : Cluster 2 . 
Figure 6

Population structure analysis of $49 \mathrm{WBPH}$ resistant accessions of common wild rice (0. rufipogon). 